\title{
Critical fronts in initiation of excitation waves
}

\author{
I. Idris and V. N. Biktashev \\ Department of Mathematical Sciences, University of Liverpool, Liverpool L69 7ZL, United Kingdom
}

(Received 1 March 2007; revised manuscript received 7 May 2007; published 7 August 2007)

\begin{abstract}
We consider the problem of initiation of propagating waves in a one-dimensional excitable fiber. In the FitzHugh-Nagumo theory, the key role is played by "critical nucleus" and "critical pulse" solutions whose (center-) stable manifold is the threshold surface separating initial conditions leading to propagation and those leading to decay. We present evidence that in cardiac excitation models, this role is played by "critical front" solutions.
\end{abstract}

DOI: 10.1103/PhysRevE.76.021906

PACS number(s): 87.19.Hh, 87.19.La, 02.90.+p

\section{INTRODUCTION}

An excitable medium is a thermodynamically nonequilibrium system that has a stable spatially uniform "resting state," but responds to an above-threshold localized stimulus by a propagating nondecaying "excitation wave." Excitation waves play key roles in living organisms and are observed in chemical and physical systems: e.g., nerves, heart muscle, catalytic redox reactions, large aspect lasers, and star formation in galaxies [1]. Understanding conditions of successful initiation of excitation waves is particularly important for the heart where such waves trigger coordinated contraction of the muscle and where a failure of initiation can cause or contribute to serious or fatal medical conditions, or render inefficient the work of pacemakers or defibrillators [2].

The theoretical understanding of excitability stems from FitzHugh's simplified model of a nerve membrane [3]. One of his key concepts is a"quasithreshold," which gets precise in the limit of large time scale separation between the processes of excitation and recovery. Then the fast subsystem has unstable "threshold" equilibria. Initial conditions below such an equilibrium lead to decay, and those above it lead to excitation.

In a spatially extended FitzHugh-Nagumo (FHN) system $[3,4]$, the ability of a stimulus to initiate a wave depends also on its spatial extent, the aspect summarized by Rushton's [5] concept of "liminal length." More generic is the concept of "critical curve" in the stimulus strength-spatial extent plane (see Fig. 1). A stimulus initiates a wave if its parameters are above this curve or leads to decay if below.

Mathematically, the problem is about classification of initial conditions that will or will not lead to a traveling-wave solution. The key question is the nature of the boundary between the two classes. A detailed analysis of this boundary has been done for the FitzHugh-Nagumo system and its variations. This has led to the concept of a critical nucleus, discussed below in more detail. Roughly, this is a spatially extended analog of a threshold equilibrium in the point system: the critical nucleus is also a stationary but unstable solution, and its small perturbations lead to either initiation of an excitation wave, for perturbations of one direction, or to decay, for perturbations of the opposite direction.

We stress that although the role of FHN as a universal prototype of excitable systems has been disputed, to our knowledge, there are still no alternatives to the critical nucleus concept, as far as the initiation problem is concerned.

In this paper, we present evidence that cardiac excitation provides an example of an alternative type of system, in which there is no place for the critical nucleus. Two independent observations led to this study. First, numerical simulations of the cardiac excitation models reveal significant qualitative differences in the way initiation occurs in such models, compared to the FHN-style systems [6]. Second, asymptotic analysis of detailed cardiac excitation models reveals that in the fast subsystem there, there is no analog of the unstable threshold equilibrium of FHN systems [7], and the threshold there has a completely different mathematical nature. Further, elementary arguments show that in cardiac equations there are no nontrivial stationary solutions that could play the role similar to the critical nucleus in FHN system.

Thus we have a theoretical vacuum here. Obviously, one cannot even begin to think about investigating initiation criteria without understanding the nature of the critical solutions. This paper aims to fill this vacuum and clarify the nature of the critical solutions. We analyze a simplified model of cardiac excitation and use the knowledge of its exact solutions to demonstrate that for this model the concept of the critical nucleus should be replaced with the concept of a critical front. We also confirm numerically the relevance of this concept on an example of a detailed ionic cardiac excitation model.

\section{FITZHUGH-NAGUMO SYSTEM}

First we recapitulate some known theoretical concepts related to initiation of waves (see, e.g., $[8,9]$ and references therein). We consider the FitzHugh-Nagumo system in the form

$$
\begin{gathered}
u_{t}=u_{x x}+f(u)-v, \\
f(u)=u(u-\theta)(1-u), \\
v_{t}=\varepsilon(\alpha u-v),
\end{gathered}
$$

where $\varepsilon>0, \alpha>0, \theta \in(0,1 / 2)$ (some works consider piecewise linear functions $f$ of similar shape) on a half-fiber, $(x, t) \in[0, \infty) \times[0, \infty)$ with a no-flux boundary, 


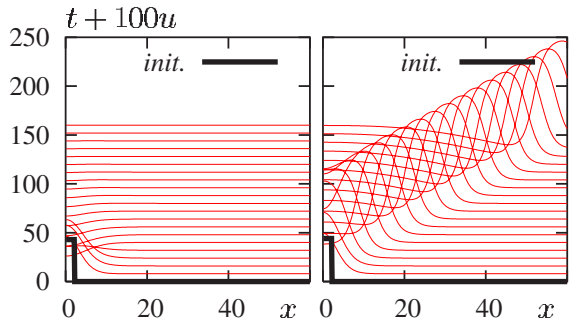

(a)

(b)

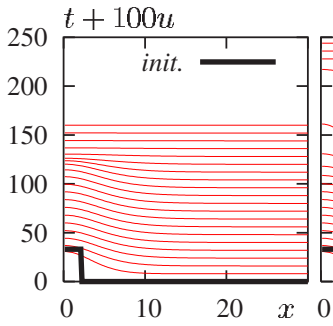

(c)

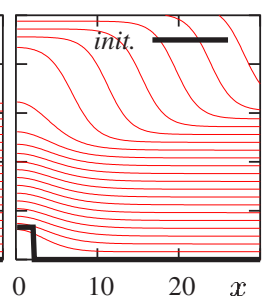

(d)

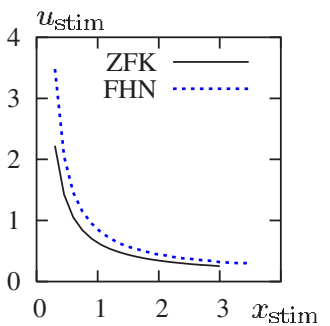

(e)

FIG. 1. (Color online) Initiation of excitation in the FitzHugh-Nagumo system. (a),(b) Full system (1)-(3) "FHN" for parameter values $\alpha=0.37, \theta=0.13$, and $\varepsilon=0.02$. Stimulation parameters: $x_{\text {stim }}=2.10$ for both below threshold $u_{\text {stim }}=0.43$, leading to decay, for (a) and above threshold $u_{\text {stim }}=0.44$, leading to initiation of excitation propagation, for (b). (c),(d) Fast subsystem (4)-(6) "ZFK": same parameters as in (a),(b) except $\varepsilon=0$. Stimulation: $x_{\text {stim }}=2.10$ for both below threshold $u_{\text {stim }}=0.3304831$ for (c) and above-threshold $u_{\text {stim }}=0.3304833$ for (d). Bold black lines: initial conditions. (e) The corresponding critical curves, separating initiation initial conditions from decay initial conditions. Simulation done on an interval $x \in[0, L], L=120$ with Neuman boundaries, central space differencing with $h_{x}=0.15$, and explicit Euler time stepping with $h_{t}=0.01$.

$$
u_{x}(0, t)=0
$$

and a rectangular initial perturbation of width $x_{\text {stim }}$ and amplitude $u_{\text {stim }}$,

$$
\begin{gathered}
u(x, 0)=u_{\text {stim }} \Theta\left(x_{\text {stim }}-x\right), \\
v(x, 0)=0,
\end{gathered}
$$

where $\Theta(\cdot)$ is the Heaviside step function.

Figures 1(a) and 1(b) show two typical results of the initiation process: a successful initiation, leading to generation of a propagating pulse, and an unsuccessful, leading to decay of excitation in the whole half-fiber into the resting state.

If $\varepsilon=0$, the problem (1) and (3) reduces to an initiation problem for the Zeldovich-Frank-Kamenetsky (ZFK) equation [10], also known as the Nagumo equation [11],

$$
\begin{gathered}
u_{t}=u_{x x}+f(u), \\
u_{x}(0, t)=0, \\
u(x, 0)=u_{\text {stim }} \Theta\left(x_{\text {stim }}-x\right) .
\end{gathered}
$$

Figures 1(c) and 1(d) illustrate the initiation and its failure in this reduced problem. Instead of a propagating pulse, successful initiation produces a propagating front. In the full model with small $\varepsilon$, this front is followed, in time scale $\mathcal{O}\left(\varepsilon^{-1}\right)$, by a wave-back to form a full excitation pulse.

A key role in understanding initiation belongs to an unstable nontrivial bounded time-independent solution of (4) and (5), sometimes called a critical nucleus, by analogy with phase transition theory. Such a solution is unique; for a cubical nonlinearity $f$ as in (1), this solution has the form $u_{\mathrm{cr}}(x)=3 \theta \sqrt{2}\left[(1+\theta) \sqrt{2}+\cosh (x \sqrt{\theta}) \sqrt{2-5 \theta+\theta^{2}}\right]^{-1}$. Its linearization spectrum has exactly one unstable eigenvalue, while all other eigenvalues are stable. So the stable manifold of this stationary solution has codimension 1 and divides the phase space of (4) and (5) to two open sets. One of these sets corresponds to initial conditions leading to successful initiation and the other to decay. In particular, if the initial condition satisfies $u(x, 0)<u_{\mathrm{cr}}(x), x \in[0, \infty)$, then $u(x, t)$ decays as $t \rightarrow \infty$, and if $u(x, 0)>u_{\mathrm{cr}}(x), x \in[0, \infty)$, then $u(x, t)$ ap- proaches a stable propagating front solution. Moreover, if a continuous one-parametric family of initial conditions contains some that initiate a wave and some that lead to decay, then there is always at least one that does neither, but gives a solution that approaches the critical nucleus. This critical nucleus is the same for all such families; e.g., it does not depend on the shape of the initial distribution $u(x, 0)$ as long as its amplitude is at the threshold corresponding to that shape. Initial conditions very close to the threshold generate solutions which approach the critical nucleus and then depart from it, either toward propagation or toward decay. This transient stationary state can be seen in Figs. 1(c) and 1(d) where the initial conditions are selected very close to the threshold.

For small $\varepsilon>0$, system (1) does not have nontrivial stationary solutions. However, for $x \in(-\infty, \infty) \mathrm{Eq}$. (1) has an unstable propagating pulse solution $\widetilde{u}_{\mathrm{cr}}(x-c t), \widetilde{v}_{\mathrm{cr}}(x-c t)$ such that $\tilde{u}_{\mathrm{cr}}(x) \rightarrow u_{\mathrm{cr}}(x), \widetilde{v}_{\mathrm{cr}}(x) \rightarrow 0$, and $c=\mathcal{O}\left(\varepsilon^{1 / 2}\right)$ as $\varepsilon \searrow 0$. Due to translational symmetry, this solution (in the comoving frame of reference) has a zero eigenvalue corresponding to the eigenfunction $\left(\tilde{u}_{\mathrm{cr}}^{\prime}, \widetilde{v}_{\mathrm{cr}}^{\prime}\right)$. This solution also has a single unstable eigenvalue. So its center-stable manifold has codimension 1 and is the threshold hypersurface dividing the phase space into the decay domain and the initiation domain. So here we have a critical pulse solution, which we define as an unstable traveling wave that is asymptotic to the resting state for both limits $x-c t \rightarrow \pm \infty$. For small $\varepsilon$, the critical pulse is essentially a slowly traveling variant of the critical nucleus. Any solution with the initial condition at the threshold hypersurface will asymptotically approach this critical pulse (suitably shifted), and any solutions starting close to the threshold will approach this critical pulse as a transient. ${ }^{1}$ This is illustrated in Fig. 2.

\footnotetext{
${ }^{1}$ The symmetry $x \leftrightarrow-x$ means there are two stable pulse solutions, one propagating to the right and one propagating to the left. Likewise, there are two critical pulse solutions, and two center-stable manifolds. The relationship between these two critical hypersurfaces is complicated, since some families of initial conditions can generate two oppositely traveling pulses and some can generate only one.
} 


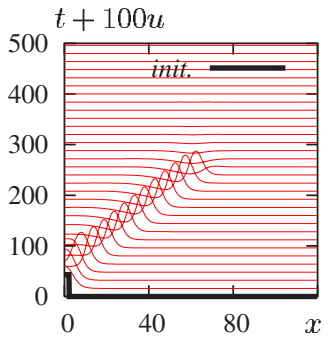

(a)

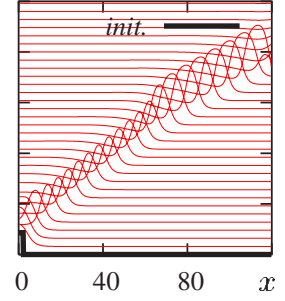

(b)

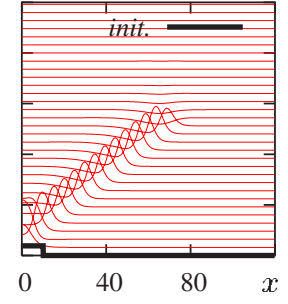

(c)

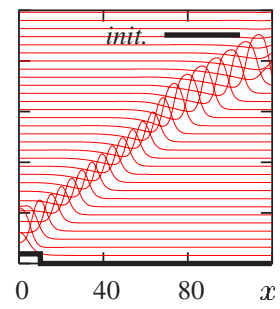

(d)

FIG. 2. (Color online) The critical pulse is a universal transient for any near-threshold initial condition. The solutions to (1) for slightly below-threshold (a),(c) and slightly above-threshold (b),(d) amplitudes, for smaller stimulus width $x_{\text {stim }}=2.10$ in (a),(b) and larger $x_{\text {stim }}$ $=10.05$ in (c),(d). Parameter values: $\varepsilon=0.02, \alpha=0.37, h_{t}=0.01, h_{x}=0.15$, and $L=120$. Stimulus amplitudes: $u_{\text {stim }}=0.431929399574766$ for (a), 0.431929399574768 for (b), 0.191802079312694 for (c), and 0.191802079312696 for (d). In all cases we see a slow, lowamplitude unstable propagating pulse which subsequently either decays or evolves into a fast, high-amplitude stable propagating pulse.

With this understanding, the excitation condition in terms of $\left(x_{\text {stim }}, u_{\text {stim }}\right)$ reduces to computing the intersection of the two-parametric manifold described by (3) with the codimension-1 stable (center-stable) manifold of the critical nucleus (critical pulse). This gives the curve on the $\left(x_{\text {stim }}, u_{\text {stim }}\right)$ plane separating initial conditions leading to excitation propagation from those leading to decay. This can be done numerically or, with appropriate simplifications, analytically. An example of dealing with this problem in the ZFK equation, using Galerkin-style approximations, can be found in [12]. Here we concentrate on the principal question of the nature of the threshold hypersurface in the functional space for cardiac excitation equations. It appears that in cardiac equations, this nature is different from the FitzHughNagumo theory just considered.

\section{SIMPLIFIED CARDIAC EXCITATION MODEL}

Now we consider the simplified model of $I_{\mathrm{Na}}$-driven excitation fronts in typical cardiac excitation models proposed in [13]:

$$
\begin{gathered}
E_{t}=E_{x x}+\Theta(E-1) h, \\
h_{t}=[\Theta(-E)-h] / \tau,
\end{gathered}
$$

with boundary condition

$$
E_{x}(0, t)=0
$$

and initial condition

$$
\begin{gathered}
E(x, 0)=-\alpha+E_{\text {stim }} \Theta\left(x_{\text {stim }}-x\right), \\
h(x, 0)=1,
\end{gathered}
$$

where the variable $E$ represents the transmembrane potential of the cardiac tissue, $h$ is the probability of the $\mathrm{Na}$ gates being open, $\tau$ is a dimensionless parameter, and $\alpha>0$ represents the prefrontal voltage, which we consider fixed in this paper. System (7) can be obtained by simplifying right-hand sides of the fast subsystem in an appropriate asymptotic limit of a typical cardiac excitation model [14]. In that sense, system (7) plays the same role for a typical cardiac excitation model as ZFK equation (4) plays for a classical activatorinhibitor excitable system like (1).
System (7) does not have nontrivial bounded stationary solutions: if $E_{t}=h_{t}=0$, then any bounded solution has the form $E=a, h=\Theta(-a), a=$ const. So there are no critical nuclei in this system. Nevertheless, system (7) gives propagating front solutions for initial conditions above a threshold and decay for those below it. Hence there is a question: what happens when the initial condition is exactly at the threshold?

System (7) has a family of propagating front solutions:

$$
\begin{gathered}
E(z)= \begin{cases}\omega-\frac{\tau^{2} c^{2}}{1+\tau c^{2}} \exp \left(\frac{z}{\tau c}\right) & (z \leq-\Delta), \\
-\alpha+\alpha \exp (-c z) & (z \geq-\Delta),\end{cases} \\
h(z)= \begin{cases}\exp \left(\frac{z}{\tau c}\right) & (z \leq 0), \\
1 & (z \geq 0),\end{cases}
\end{gathered}
$$

where $z=x-c t, \omega=1+\tau c^{2}(\alpha+1)$, and $\Delta=\frac{1}{c} \ln \left(\frac{1+\alpha}{\alpha}\right)$ and parameters $c, \alpha$, and $\tau$ are related by

$$
\tau c^{2} \ln \left[(1+\alpha)\left(c^{2}+\tau^{-1}\right)\right]+\ln \left(1+\alpha^{-1}\right)=0 .
$$

For a fixed $\alpha$, there is a $\tau_{*}(\alpha)$ such that for $\tau>\tau_{*}$, Eq. (11) has two solutions for $c: c=c_{ \pm}(\alpha, \tau)$ and $c_{+}>c_{-}[13]$. There is numerical and analytical evidence that solutions with $c=c_{+}$ are stable and those with $c=c_{-}$are unstable with one positive eigenvalue $[13,15]$.

Hence by analogy with the FHN system, we propose the following.

Conjecture 1. The center-stable manifold of the unstable front solution (10) with $c=c_{-}(\alpha, \tau)$ is the threshold hypersurface, separating the initial conditions leading to initiation from the initial conditions leading to decay. ${ }^{2}$

That is, instead of a critical nucleus or a critical pulse solution, the role of the threshold solution is played by a "critical front," which we define as a traveling-wave solution with different asymptotics at $x-c t \rightarrow+\infty$ and $x-c t \rightarrow-\infty$ : the prefrontal state and the postfrontal state.

\footnotetext{
${ }^{2}$ Again, symmetry $x \leftrightarrow-x$ implies there are actually two hypersurfaces, partly connected with each other.
} 

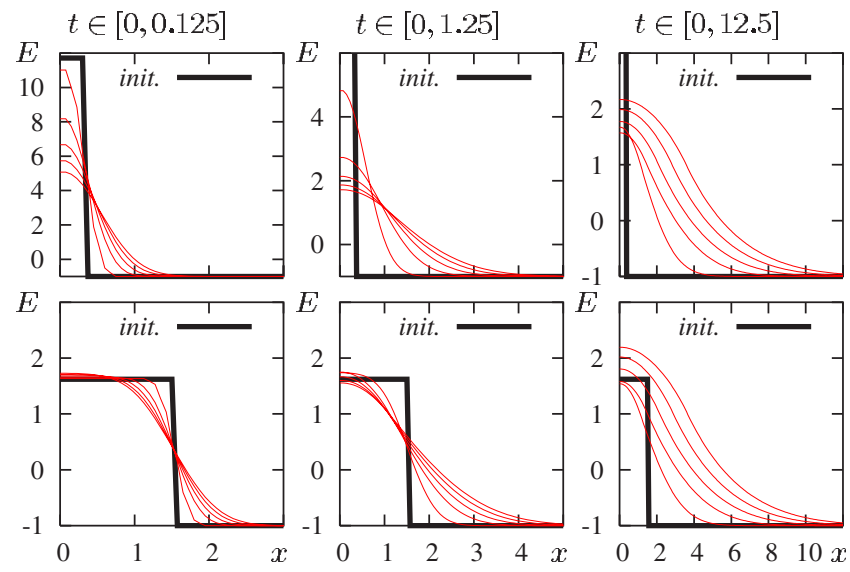

FIG. 3. (Color online) Evolution of two different near-threshold initial conditions toward the critical front solution in system (7). Initial stimuli: $x_{\text {stim }}=0.3, E_{\text {stim }}=12.716330706144868$ (upper row) and $x_{\text {stim }}=1.5, E_{\text {stim }}=2.619968799545055$ (lower row). Other parameters: $\tau=8.2, \alpha=1, h_{x}=0.075, h_{t}=0.0025$, and $L=50$.

An "experimentally testable" consequence of this conjecture is that for any initial condition exactly at the threshold, the solution will approach the unstable front as $t \rightarrow+\infty$. For any initial condition near the threshold, the solution will come close to the unstable front and stay in its vicinity for a long time: if the positive eigenvalue is $\lambda$ and the initial condition is $\delta$, close to the threshold, the transient front should be observed for time of the order of $\lambda^{-1}|\ln \delta|$. This transient front solution will not depend on the initial condition, as long as the initial condition is at the threshold.

We have tested these predictions by numerical simulation of (7)-(9). The results are shown in Figs. 3 and 4.

Figure 3 illustrates two solutions starting from initial conditions with different $x_{\text {stim }}$ values. In both cases, $E_{\text {stim }}$ values
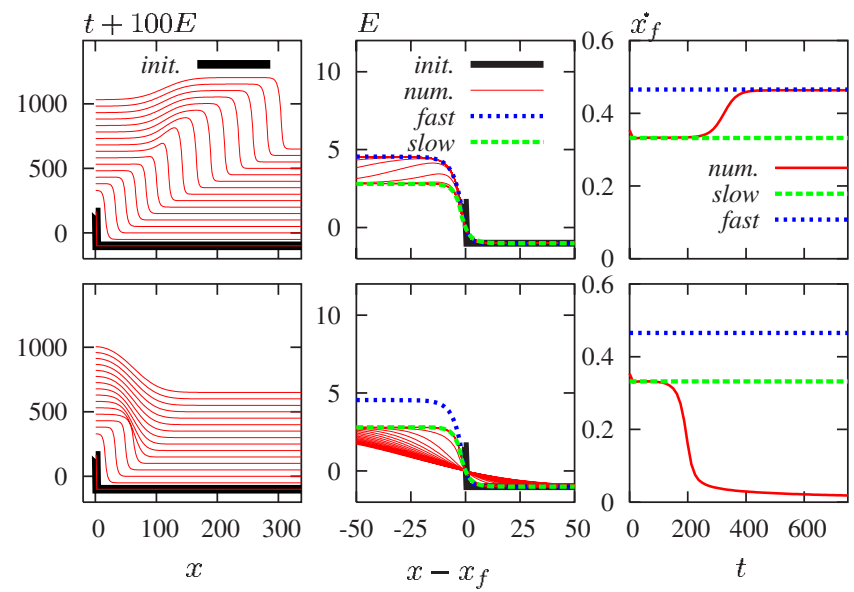

FIG. 4. (Color online) Transient critical fronts are close to the unstable front solution of (7). Initial conditions: $x_{\text {stim }}=1.5$, with $E_{\text {stim }}=2.619968799545055$ in the upper row and $E_{\text {stim }}$ $=2.619968799545054$ in the lower row, other parameters the same as in Fig. 3. Left column: evolution of the $E$ profiles in the laboratory frame of reference. Middle column: same evolution, in the frame of reference comoving with the front. Right column: speed of the front. Blue (green) dashed lines in the middle and right columns correspond to the exact fast (slow) front solutions of (7). have been chosen close to the respective thresholds with high precision. In both cases, the solutions evolve in the long run toward the same propagating front.

Figure 4 presents an analysis of a pair of solutions, one with slightly above-threshold and the other with slightly below-threshold initial conditions. To separate the evolution of the front shape from its movement, we employed the idea of symmetry group decomposition with explicit representation of the orbit manifold (see, e.g., [16]). Practically, we define the front point $x_{f}=x_{f}(t)$ via

$$
E\left(x_{f}(t), t\right)=E_{*}
$$

for some constant $E_{*}$ which is guaranteed to be represented exactly once in the front at every instant of time (we have chosen $\left.E_{*}=0\right)$. Then $E\left(x-x_{f}(t), t\right)$ gives the voltage profile "in the standard position" and $x_{f}(t)$ describes the movement of this profile.

The predictions based on conjecture 1 are that the voltage profile should, after an initial transient depending on the initial condition, approach the profile of the slow unstable front solution given by $(10)$ with $c=c_{-}(\tau, \alpha)$ and stay close to it for some time, before either developing into the fast stable front (10) with $c=c_{+}(\tau, \alpha)$ or decaying. Likewise, the speed of the front should, after an initial transient, be close to the speed of the slow unstable front $c_{-}(\tau, \alpha)$, before either switching the speed of the fast stable front $c_{+}(\tau, \alpha)$ or dropping to zero. This is precisely what is seen in Fig. 4, where we have taken advantage of knowing the exact solutions $E\left(x-c_{ \pm} t\right)$ and $c_{ \pm}$ for both the fast and slow fronts.

Initial conditions with different $x_{\text {stim }}$ and $E_{\text {stim }}$ close to the corresponding threshold produce the same picture with the exception of the initial transient. We have also checked that the length of the time period during which the solution stays close to the unstable front is, roughly, a linear function of the number of correct decimal figures in $E_{\text {stim }}$, as it should be according to conjecture 1 .

\section{DETAILED CARDIAC EXCITATION MODEL}

The simplified model (7) is quantitatively very far from any realistic ionic model of cardiac excitation and has many peculiar qualitative features stemming from the nonstandard asymptotic embedding leading to it. Hence the newly described phenomenon of critical front could be an artifact of the simplifications. To eliminate this possibility, we have tested the relevance of the critical front concept to a full ionic model of cardiac excitation. We have chosen the model of human atrial tissue due to Courtemanche, Ramirez, and Nattel (CRN) [17], which is less stiff than a typical ventricular or Purkinje fiber model, is well formulated in a mathematical sense, and is popular among cardiac modelers. This model operates with 21 dynamic variables including the transmembrane voltage $V$. We have used the default parameter values as described in [17] and supplemented the equation for $V$ in the ordinary differential equations system with a diffusion term $D \partial^{2} V / \partial x^{2}$. As the spatial scale is not important for the question at hand, we assumed $D=1$. The initial conditions for $V$ were taken in the form 


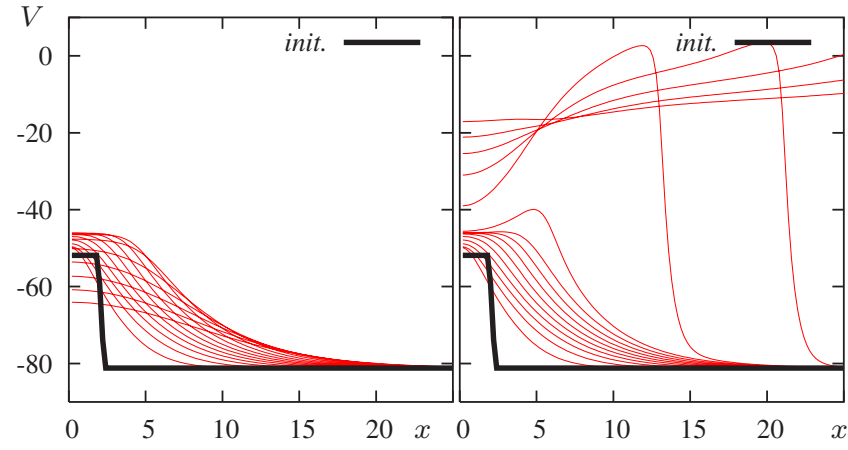

FIG. 5. (Color online) Critical fronts in the CRN model [17]. Shown are voltage profiles in every $10 \mathrm{~ms}$. Parameter values: $h_{t}=0.01 \mathrm{~ms}, h_{x}=0.2$, and $L=40$, the length unit chosen so that voltage diffusion coefficient equals 1 . Stimulus width $x_{\text {stim }}=2$, stimulus amplitudes: $\quad V_{\text {stim }}=29.31542299307152 \mathrm{mV}$ (left panel) and $V_{\text {stim }}=29.31542299307153 \mathrm{mV}$ (right panel). The critical fronts are formed within the first $10 \mathrm{~ms}$ and then are seen for the subsequent $80 \mathrm{~ms}$ on both panels before exploding into an excitation wave of much larger amplitude and speed in the right panel and decaying in the left panel.

$$
V(x, 0)=V_{r}+V_{\text {stim }} \Theta\left(x_{\text {stim }}-x\right),
$$

where $V_{r}=-81.18 \mathrm{mV}$ is the standard resting potential, and for all other 20 variables at their resting values as described in [17]. Figure 5 illustrates a pair of solutions with initial conditions slightly above and slightly below the threshold. The critical front solution is clearly seen there: it has the upper voltage of about $-46 \mathrm{mV}$ and during $80 \mathrm{~ms}$ of its existence propagates with a speed approximately 0.06 space units per millisecond. Then for the above-critical case it develops into an excitation front with maximal voltage about $+3 \mathrm{mV}$ and speed 0.8 space units per millisecond, and decays for the below-critical case.

Mathematically, the post-front voltage of about $-46 \mathrm{mV}$ observed in Fig. 5 is not a true equilibrium of the full CRN model, so the critical front can only be an asymptotic concept in an appropriate asymptotic embedding, say as ones described in [14] or [18], and the observed critical front may well be the front of a critical pulse solution in the full model. However Fig. 5 demonstrates that the critical front is a practical and well-working concept even for the full model, unlike the critical pulse, which may be theoretically existing, but practically unobservable: notice the number of significant decimal digits in initial conditions required to produce only the critical front observed for $80 \mathrm{~ms}$ and recall that the number of decimals is roughly proportional to the duration of the observation of an unstable solution.

\section{DISCUSSION}

We have presented numerical evidence that the centerstable manifold of the unstable slow front solution of the fast subsystem of a cardiac excitation model serves as the threshold hypersurface separating initial conditions leading to successful initiation and those leading to decay. This means, e.g., that a critical curve for a two-parametric family of initial conditions, be that family (9) with parameters $\left(x_{\text {stim }}, E_{\text {stim }}\right)$ or any other, can be found as an intersection of this codimension-1 critical hypersurface with the twodimensional manifold of those initial conditions. Finding this hypersurface can be done numerically or analytically using suitable approximation-e.g., as was done in [12,19] for the ZFK equation; this is a subject for further investigation.

Another problem for future study is to verify that the findings remain qualitatively true for formal asymptotic embeddings of various cardiac excitation models and, in particular, which mathematical features of these embeddings are essential for the existence of the critical fronts. Of principal importance is the conclusion that for cardiac equations, instead of the "critical nucleus" or its slowly moving variant "critical pulse" known from the FitzHugh-Nagumo theory, we now have a "critical front solution." This, in particular, means physically that the make-or-break conditions of cardiac excitation waves are restricted to a vicinity of their fronts.

Finally, we believe that this study sets a useful example for initiation problems in other types of excitable systems, alternative to the existing critical nucleus theory, since not all, if any, real-world excitable systems are well described by an asymptotic structure as in (1).

\section{ACKNOWLEDGMENTS}

The authors are grateful to C.F. Starmer and E.E. Shnol for inspiring discussions. This study has been supported by EPSRC Grant No. GR/S75314/01 and MacArthur Foundation Grant No. 71356-01.
[1] Wave and Patterns in Biological and Chemical Excitable Media, edited by V. Krinsky and H. Swinney (North-Holland, Amsterdam, 1991).

[2] Cardiac Electrophysiology: From Cell to Bedside, edited by D. P. Zipes and J. Jalife (Saunders, Philadelphia, 2000).

[3] R. FitzHugh, Biophys. J. 1, 445 (1961).

[4] J. Nagumo, S. Arimoto, and S. Yoshizawa, Proc. IRE 50, 2061 (1962).

[5] W. Rushton, Proc. R. Soc. London, Ser. B 124, 210 (1937).

[6] C. F. Starmer, A. O. Grant, and T. J. Colatsky, Cardiovasc.
Res. 57, 1062 (2003)

[7] V. N. Biktashev and R. Suckley, Phys. Rev. Lett. 93, 168103 (2004).

[8] G. Flores, J. Diff. Eqns. 80, 306 (1989).

[9] G. Flores, SIAM J. Math. Anal. 22, 392 (1991).

[10] Y. B. Zel'dovich, D. A. Frank-Kamenetsky, and A. N. Doklady, Dokl. Akad. Nauk SSSR 19, 693 (1938).

[11] H. P. McKean, Jr., Adv. Appl. Math. 4, 209 (1970).

[12] J. C. Neu, R. S. Preissig, and W. Krassowska, Physica D 102, 285 (1997). 
[13] V. N. Biktashev, Phys. Rev. Lett. 89, 168102 (2002).

[14] I. V. Biktasheva, R. D. Simitev, R. S. Suckley, and V. N. Biktashev, Philos. Trans. R. Soc. London, Ser. A 364, 1283 (2006).

[15] R. Hinch, Bull. Math. Biol. 66, 1887 (2004).

[16] V. N. Biktashev and A. V. Holden, Physica D 116, 342 (1998).

[17] M. Courtemanche, R. J. Ramirez, and S. Nattel, Am. J.
Physiol. 275, H301 (1998).

[18] R. D. Simitev and V. N. Biktashev, Biophys. J. 90, 2258 (2006).

[19] V. Moll and S. I. Rosencrans, SIAM J. Appl. Math. 50, 1419 (1990). 